\title{
Investigating the use of a hybrid plasmonic-photonic nanoresonator for optical trapping using finite-difference time-domain method
}

\author{
M. Mossayebi ${ }^{1}$ - A. J. Wright ${ }^{1} \cdot$ A. Parini ${ }^{2}$ M. G. Somekh ${ }^{1,3}$ • \\ G. Bellanca ${ }^{2}$ E. C. Larkins ${ }^{1}$
}

Received: 4 August 2015/ Accepted: 22 March 2016/Published online: 8 April 2016

(C) The Author(s) 2016. This article is published with open access at Springerlink.com

\begin{abstract}
We investigate the use of a hybrid nanoresonator comprising a photonic crystal $(\mathrm{PhC})$ cavity coupled to a plasmonic bowtie nanoantenna (BNA) for the optical trapping of nanoparticles in water. Using finite-difference time-domain simulations, we show that this structure can confine light to an extremely small volume of $\sim 30,000 \mathrm{~nm}^{3}(\sim 30 \mathrm{zl})$ in the BNA gap whilst maintaining a high quality factor (5400-7700). The optical intensity inside the BNA gap is enhanced by a factor larger than 40 compared to when the BNA is not present above the $\mathrm{PhC}$ cavity. Such a device has potential applications in optical manipulation, creating high precision optical traps with an intensity gradient over a distance much smaller than the diffraction limit, potentially allowing objects to be confined to much smaller volumes and making it ideal for optical trapping of Rayleigh particles (particles much smaller than the wavelength of light).
\end{abstract}

Keywords Optical trapping · Optical tweezers · Plasmonics · Photonics · Nanoplasmonics · Nanophotonics

This article is part of the Topical Collection on Optical Wave \& Waveguide Theory and Numerical Modelling, OWTNM' 15.

Guest edited by Arti Agrawal, B.M.A. Rahman, Tong Sun, Gregory Wurtz, Anibal Fernandez and James R. Taylor.

M. Mossayebi

eexmm30@nottingham.ac.uk

1 Electrical Systems and Optics Division, University of Nottingham, Nottingham, UK

2 Department of Engineering, University of Ferrara, Ferrara, Italy

3 Department of Electronic and Information Engineering, Hong Kong Polytechnic University, Kowloon, Hong Kong, China 


\section{Introduction}

In the past 30 years, optical trapping and manipulation (Ashkin 1970; Ashkin et al. 1986; Grier 2003; Neuman and Block 2004; Molloy and Padgett 2002; Dienerowitz et al. 2008; Marag et al. 2013; Ashkin 1997) has been a major tool for advances in biosciences (Khatibzadeh et al. 2014), physics (Gustavson et al. 2001) and chemistry (Misawa et al. 1991). In general, optical tweezers use forces based on the radiation pressure of laser light as a means to immobilise, rotate (Friese et al. 1998), Deufel et al. (2007) and guide small particles ranging from tens of nanometers to tens of micrometers in size (Grier 2003; Dienerowitz et al. 2008; Marag et al. 2013). They have been used in cutting edge research where small particles play a role. The applications of optical trapping include: the observation of the angular momentum of light (Yao and Padgett 2011), the transport of Bose-Einstein condensates (Gustavson et al. 2001), the investigation of the DNA mechanics (Bustamante et al. 2003), spectroscopy and sensing (Cetin et al. 2011), cancer research (Cross et al. 2007), nanofabrication (Pauzauskie et al. 2006), tissue engineering (Kim et al. 1999; Matsuda and Sugawara 1996), the manipulation of single proteins (Pang and Gordon 2012) and single atoms (Yu et al. 2014) and study and manipulation of live cell dynamics in animals (Zhong et al. 2013).

Conventional optical tweezers are most commonly used to trap particles in the Mie regime, where the particle size is of the order of the wavelength of light. In the Rayleigh regime, however, where the particle size is considerably smaller than the wavelength of light, the mechanisms to generate the optical forces rely more heavily on the material properties of the particle. This means that the optical trapping forces are typically lower, and greater power is often required to enable stable trapping. This can in turn cause damage to thel specimen in the trap. In this regime, it is particularly difficult to trap transparent biological objects with relatively low dielectric contrast (Juan et al. 2011; Dholakia and Reece 2006). Svoboda and Block (1994) reported that the gradient force is the major trapping force for Rayleigh sized particles and is proportional to their polarizability. They reported that it is $7 \times$ easier to trap $36 \mathrm{~nm}$ gold nanoparticles compared to 38 $\mathrm{nm}$ latex beads - a factor equal to the ratio of the polarizabilities of the particles. For the same optical illumination, this results in higher trapping forces for metallic nanoparticles than for dielectric nanoparticles.

Near field optical tweezers use plasmonic substrates (Min et al. 2013) such as nanoantenna pairs (Grigorenko et al. 2008; Cetin et al. 2011; Righini et al. 2009; Ko et al. 2010; Roxworthy et al. 2012; Zhang et al. 2010; Wong et al. 2011) and plasmonic nanocavities (Chen et al. 2012) to confine and enhance the optical field in a subwavelength "hotspot", thereby creating stable traps for nanoparticles. However, these plasmonic devices have relatively low quality factors (less than 100) due to the radiative and absorption losses of the metal structures, making them inefficient in terms of input power (Bozhevolnyi et al. 2006). Enhanced optical tweezers using photonic devices (Mandal et al. 2010; Cai and Poon 2010; Chen et al. 2012) such as microring resonators (Lin et al. 2010; Lin and Crozier 2011, coupled nanobeam cavities (Renaut et al. 2013), optical fibers (Liberale et al. 2013) $\mathrm{PhC}$ waveguides (Yu et al. 2014) and cavities (Van Leest and Caro 2013) have also been demonstrated. PhC resonators have high quality factors ( $\mathrm{Q} \sim 500$ $100,000)$ and enable enhancement and control of the profile of the field (Lin and Crozier 2011), but they have lower spatial confinement (typically $0.5-10 \mu \mathrm{m}$ ) compared to near field plasmonic optical traps, which produce field hotspots of $\sim$ tens of nanometers (Schuller et al. 2010). 
Recently, there have been efforts to demonstrate combined photonic and plasmonic devices. Hybrid plasmonic-photonic devices were reported in 2014 for lasing (Zhang et al. 2014) and light matter coupling (Michael et al. 2010). In 2014, ODell et al. reported a technique for assembling photonic-plasmonic nanotweezers by optically guiding multiwalled carbon nanotubes to attach them onto a silicon waveguide (ODell et al. 2014). In the same year Berthelot et al. demonstrated a 3D near-field nanotweezer capable of trapping nanoparticles by fabricating a bowtie aperture on the end of a tapered, metal coated optical fiber (Berthelot et al. 2013).

In this paper, we propose to integrate photonic and plasmonic nanoresonators to take advantage of the high quality factor of the photonic cavity and the extreme light confinement of the plasmonic resonator for the optical trapping of Rayleigh particles in water. This device will have applications in biology, chemistary, naotechnology and quantum physics. In Mossayebi et al. (2016), we reported the design parameters for an L3 PhC coupled to a gold BNA separated by a silicon dioxide $\left(\mathrm{SiO}_{2}\right)$ layer, where the structure was suspended in air. In this paper, we investigate the suitability of this device for the optical trapping of biological samples. For this application, we look at the performance of the device when it is immersed in water and introduce a polystyrene (PS) sphere above the BNA gap to represent the trapped particle. Finally, we also look at the gradient profile of the optical intensity, which is a key parameter for producing a stable optical trap. This device will sit on a substrate beneath the sample of interest replacing the standard coverslip, making it compatible with the use of an inverted microscope and commonly used sample preparation methods. We show that this hybrid cavity has the ability to confine light into extremely small volumes (outside the $\mathrm{PhC}$ and in the BNA gap) whilst maintaining a high quality factor.

\section{Description of the structure and the FDTD model}

\subsection{Description of the structure}

The hybrid plasmonic-photonic nanostructure proposed in this paper is illustrated in Fig. 1. The structure consists of an $L 3 \mathrm{PhC}$ cavity based on a $275 \mathrm{~nm}$ thick gallium arsenide (GaAs) slab, a $40 \mathrm{~nm}$ thick gold (Au) BNA and a $175 \mathrm{~nm}$ thick $\mathrm{SiO}_{2}$ layer separating the two and keeping the biological sample out of contact with the GaAs membrane. The $\mathrm{PhC}$ has a triangular lattice of holes with a lattice constant, $a$, of $410 \mathrm{~nm}$ and a hole radius, $r$, of $125 \mathrm{~nm}$. The $\mathrm{L} 3$ cavity is created by removing three adjacent holes in the lattice. The end holes of the cavity are shifted by $0.2 \mathrm{a}$ to improve the quality factor of the PhC cavity (Malaguti et al. 2013; Sauvan et al. 2005). The holes of the PhC extend through the $\mathrm{SiO}_{2}$ layer to preserve the high quality factor of the structure. The BNA consists of two head to head isosceles triangles placed $30 \mathrm{~nm}$ apart (BNA gap), with a tip width of $30 \mathrm{~nm}$ and an aperture angle of 30 degrees. The length of the BNA (including the gap) is $700 \mathrm{~nm}$ and is oriented perpendicular to the long axis (X direction) of the $L 3$ cavity. The hybrid structure is immersed in water and a PS bead with a $50 \mathrm{~nm}$ radius is positioned $45 \mathrm{~nm}$ above the $\mathrm{SiO}_{2}$ membrane to represent a particle in the trap. Further details of these specific design parameters and dimensions can be found in (Mossayebi et al. 2016).

\subsection{Description of the FDTD model}

The simulation results presented in this paper were produced using 3D-FDTD calculations (Taflove and Hagness 2005), namely the Lumerical and Meep packages, with a conformal 


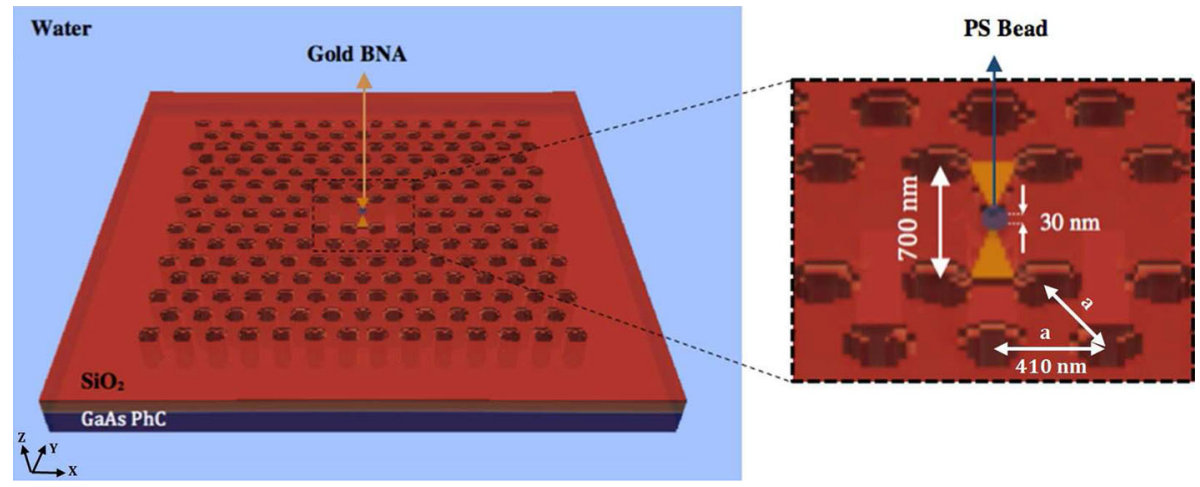

Fig. $13 \mathrm{D}$ schematic of the hybrid photonic-plasmonic nano cavity showing the dimensions of the structure. The strcuture consists of an L3 PhC cavity based on a $275 \mathrm{~nm}$ thick GaAs slab, a $40 \mathrm{~nm}$ thick Au BNA and a $175 \mathrm{~nm}$ thick $\mathrm{SiO}_{2}$ layer separating the two. The $\mathrm{PhC}$ has a triangular lattice of air holes with a lattice constant, a, of $410 \mathrm{~nm}$, and hole radius, r, of $125 \mathrm{~nm}$. The holes of the $\mathrm{PhC}$ extend through the $\mathrm{SiO}_{2}$ layer. The BNA has a length of $700 \mathrm{~nm}$ and a gap size of $30 \mathrm{~nm}$

mesh in $\mathrm{x}, \mathrm{y}$ and $\mathrm{z}$ (i.e. a varying mesh size depending on sample dimensions) and a minimum step size of $0.25 \mathrm{~nm}$ in the mesh grid (in $\mathrm{x}, \mathrm{y}$ and $\mathrm{Z}$ directions). A $500 \mathrm{~nm}$ perfectly matched layer (PML) was placed at the boundaries of the computational domain to prevent artifacts due to numerical reflections. A Drude-Lorentz model (Palik parameters Palik 2012) was used to represent the real metal behaviour of the BNA (i.e. skin effect, dispersion etc.).

A TE polarized field was produced using an $H_{z}$ dipole providing a Gaussian temporal pulse to excite the hybrid structure and investigate its resonances and their quality factors. The source was turned on for approximately $300 \mathrm{ps}$ and the simulation was run for approximately 3000 ps to ensure that the signal fully decayed by the end of the simulation and the results were accurate. The time step in the simulation was chosen to be small ( $\delta \mathrm{t}=0.267 \mathrm{ps})$ to ensure accurate results. The dipole was positioned near the center of the $\mathrm{PhC}$ cavity to excite all of the modes of the resonator. Dipole probes were used to capture the optical field at the center of the $\mathrm{BNA}, \mathrm{SiO}_{2}$ layer and the $\mathrm{PhC}$ cavity to measure the quality factor of the structure. The field profiles were captured by performing a discrete Fourier transform (DFT) on the electromagnetic field components at resonance.

\section{Characteristics of the hybrid plasmonic-photonic nanostructure in the presence of water (simulation results)}

We investigated the suitability of the hybrid device for the optical trapping of Rayleigh particles by building on the previous model. In particular, we surrounded the device with water (as opposed to air) and introducing a PS bead above the BNA gap to represent a trapped object.

\subsection{Resonant modes in water}

The quality factor, $\mathrm{Q}$, is an important design parameter of nanophotonic structures. It is defined as the total energy stored devided by energy lost per radian (Eq. 1) and determines 
the photon life time (and the enhancement in the stored energy for a given input power) in these structures.

$$
Q=\frac{2 \pi v_{0} \omega(t)}{-d \omega(t) / d t},
$$

where $v_{0}$ is the oscillation frequency and $\omega(t)$ is the energy stored in the mode at time $\mathrm{t}$.

Table 1 lists the resonant wavelengths and quality factors of the first three transverse electric (TE) modes for different configurations of the structure suspended in water and in air for comparison. Figure 2 shows the mode profiles of the first three TE modes of the L3 cavity in an XY plane passing through the center of the cavity in water. Note that the maximum of the field is in the plane passing through the center of the PhC cavity. In the next section, we show how this field couples to the BNA to produce a localized "hotspot" in the BNA gap.

Unlike in air (Mossayebi et al. 2016), the introduction of the BNA decreases the quality factor of the PhC cavity from 8200 to 5495 for the first resonant mode. We believe this is due to the reduction of the refractive index contrast between the surrounding medium and the $\mathrm{PhC}$ and a corresponding change in the $\mathrm{PhC}$ bandgap energy. Nevertheless, the structure still produces an impressively high quality factor compared to a simple near field plasmonic optical tweezer $(\mathrm{Q}<100)$. Interestingly, when a particle is positioned above the BNA gap, the quality factor of the hybrid device increases from 5495 to 7726 as the bead acts as a resonator to reduce the radiative loss of the system. With the BNA and bead present, the quality factor is only $5.8 \%$ smaller than the original $\mathrm{PhC}$ cavity submerged in water and $36.7 \%$ smaller than the hybrid structure in air.

\subsection{Confinement and enhancement of the optical field}

Figure 3 illustrates the field profiles in an yz plane passing through the center of the hybrid structure submerged in water for the cases where: only the $\mathrm{PhC}$ and the $\mathrm{SiO}_{2}$ layers are present (Top); the BNA is also present (Center); and where both the BNA and the PS bead are present (bottom). Figure 4 shows the optical intensity in the presence and absence of the BNA in the $\mathrm{x}, \mathrm{y}$ and $\mathrm{z}$ axes passing through the center of the BNA gap.

Table 1 Resonant wavelengths and quality factors of the first three TE modes of the structure with and without the BNA and with and without the polystyrene bead both in water and air (Mossayebi et al. 2016)

\begin{tabular}{|c|c|c|c|c|c|c|}
\hline \multirow[t]{2}{*}{ Structure } & \multicolumn{2}{|c|}{$\begin{array}{l}\text { First resonant } \\
\text { mode }\end{array}$} & \multicolumn{2}{|c|}{$\begin{array}{l}\text { Second } \\
\text { resonant } \\
\text { mode }\end{array}$} & \multicolumn{2}{|c|}{$\begin{array}{l}\text { Third } \\
\text { resonant } \\
\text { mode }\end{array}$} \\
\hline & $\lambda(\mathrm{nm})$ & Q & $\lambda(\mathrm{nm})$ & Q & $\lambda(\mathrm{nm})$ & Q \\
\hline $\mathrm{PhC}, \mathrm{L} 3+\mathrm{SiO}_{2}$ in air (Mossayebi et al. 2016) & 1554 & 11672 & 1484 & 1920 & 1368 & 1031 \\
\hline $\mathrm{PhC}, \mathrm{L} 3+\mathrm{SiO}_{2}+\mathrm{BNA}$ in air (Mossayebi et al. 2016) & 1555 & 12210 & 1484 & 1915 & 1368 & 1829 \\
\hline $\mathrm{PhC}, \mathrm{L} 3+\mathrm{SiO}_{2}$ in water & 1593 & 8200 & 1522 & 700 & 1408 & 1270 \\
\hline $\mathrm{PhC}, \mathrm{L} 3+\mathrm{SiO}_{2}+\mathrm{BNA}$ in water & 1591 & 5495 & 1520 & 716 & 1405 & 1270 \\
\hline $\mathrm{PhC}, \mathrm{L} 3+\mathrm{SiO}_{2}+\mathrm{BNA}+\mathrm{PS}$ bead in water & 1591 & 7726 & 1520 & 709 & 1405 & 1255 \\
\hline
\end{tabular}


Fig. 2 Plots of the mode patterns (|E|, log scale with dark blue corresponding to -3 and red corresponding to 0.5 ) of the three resonant TE modes of the L3 cavity in an $\mathrm{X} Y$ plane passing through the center of the resonator in water. Each plot is normalised with respect to its maximum. Top first resonant mode at $\lambda=1.593 \mu \mathrm{m}$. Center second resonant mode at $\lambda=1.522 \mu \mathrm{m}$. Bottom third resonant mode at $\lambda=1.408 \mu \mathrm{m}$. (Color figure online)
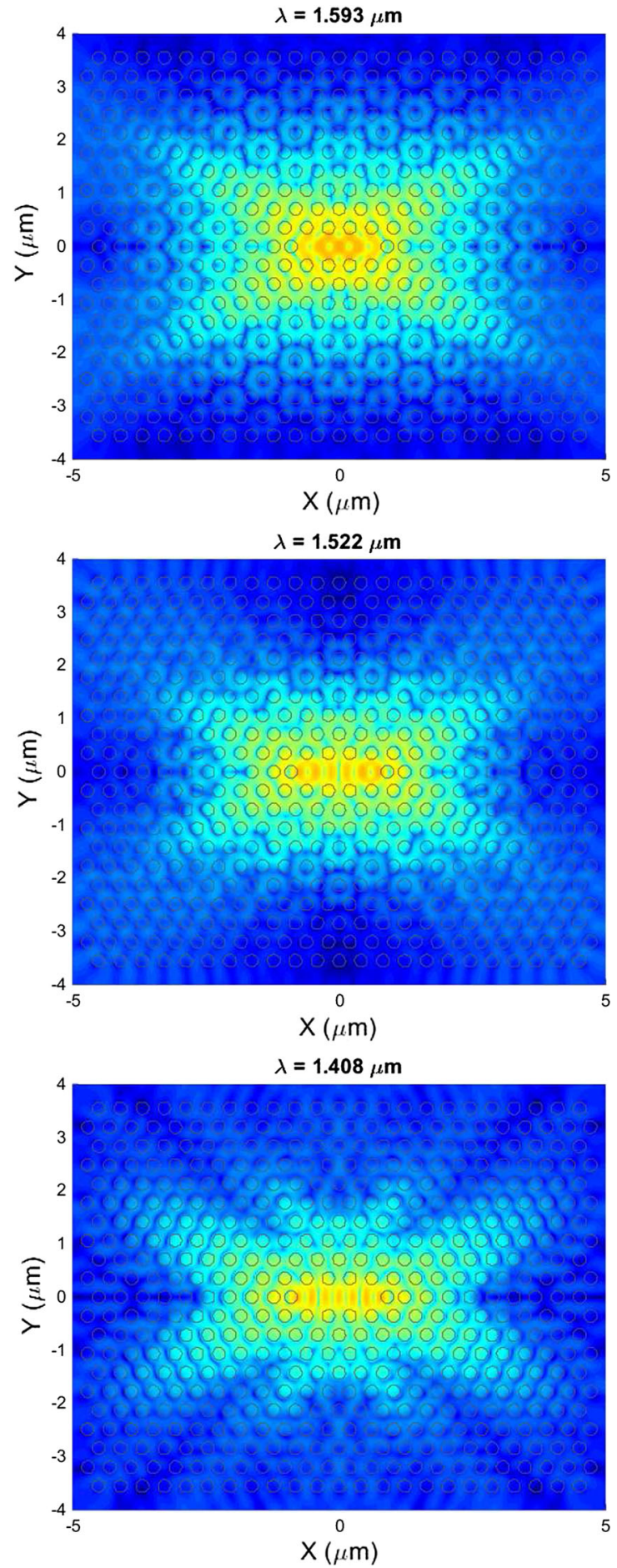

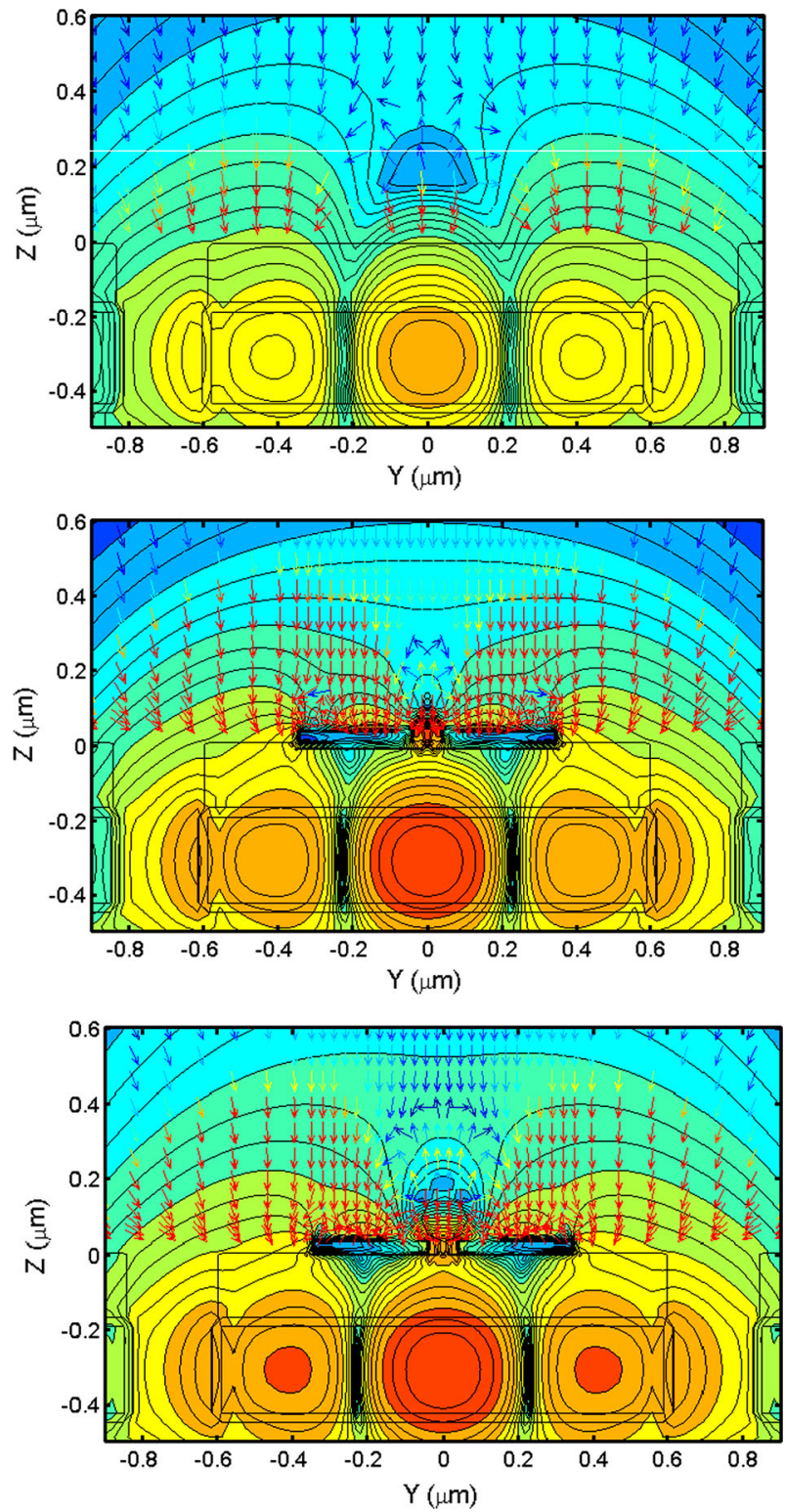

Fig. 3 The optical intensity pattern $\left(|E|^{2}\right.$, log scale) inside a YZ slice passing through the middle of the structure suspended in water: in absence of both the BNA and PS bead (Top); in the presence of the BNA (Center); and in the presence of both the BNA and PS bead (Bottom). These patterns are for the fundamental TE mode of the structure. The arrows show the field gradients and are colour coded so that the red represents the highest intensity gradient and light blue represents the lowest intensity gradient. Each graph is independently normalised with respect to its maximum field. All of the structures where excited with the same power. Dark blue represents 0.146 in the top figure, 0.135 in the center figure and 0.133 in the bottom figure. Red represents 0.428 in the top figure, 0.14 in the center figure and 0.137 in the bottom figure. (Color figure online) 
Fig. 4 Illustration of the optical intensity in the structure with (blue curve with a marker) and without (red curve) the BNA. Top intensity profile of the mode along the $\mathrm{x}$ axis passing through the center of the BNA. Center intensity profile of the fundamental mode along the $y$ axis passing through the center of the BNA. Bottom intensity profile of the fundamental mode along the $\mathrm{z}$ axis passing through the center of the BNA. All of the structures have the same excitation power. (Color figure online)
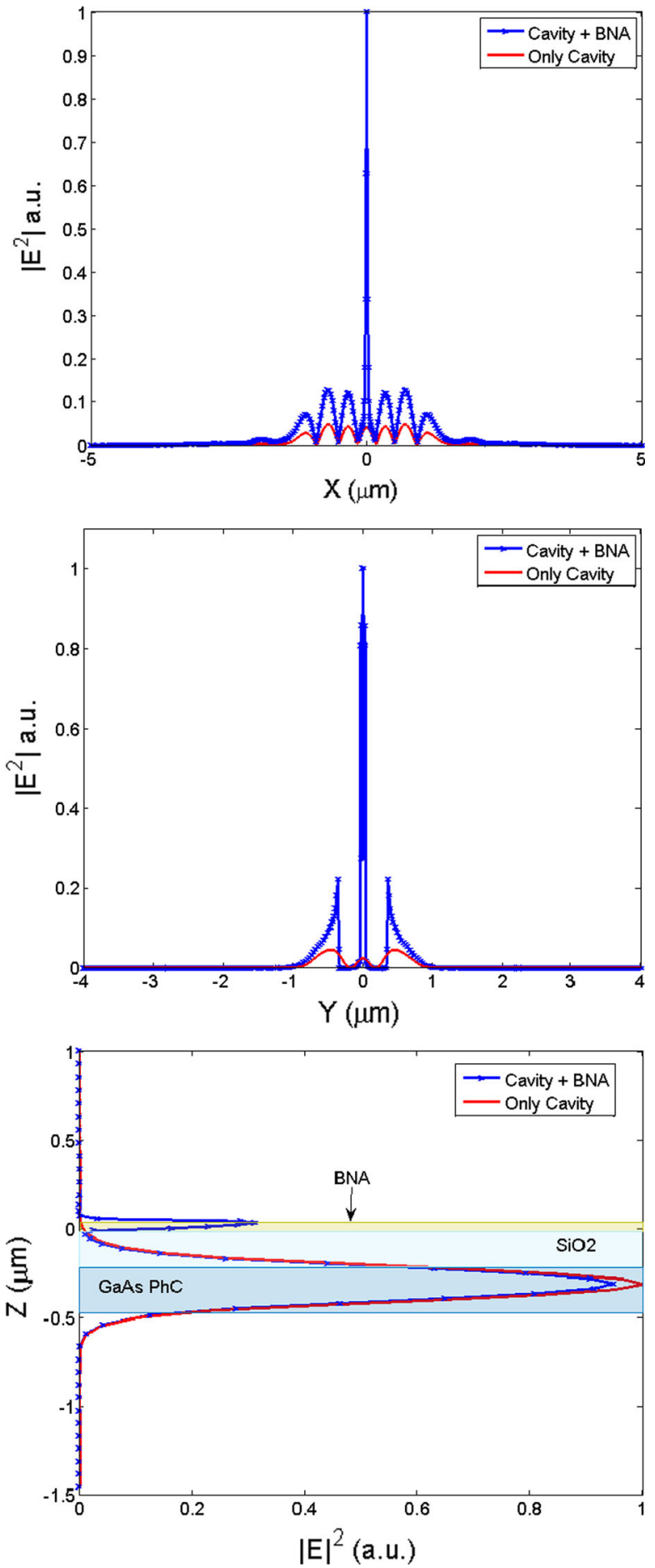
Figure 3 shows that the field is coupled from the PhC cavity to the BNA gap to produce an externally accessible "hot spot". This is confirmed in Fig. 4, where it can be seen that the optical intensity in the BNA gap is enhanced by a factor greater than 40 by introducing the BNA to the structure. This leads to an enhanced optical intensity gradient at the center of the BNA gap, which is proportional to the gradient force in optical trapping and will increase the trap strength. The field is confined to the BNA gap to a volume $\sim 30 \mathrm{~nm} \times 30 \mathrm{~nm} \times 40 \mathrm{~nm}(\mathrm{x}, \mathrm{y}, \mathrm{z})$, or $\sim 30 \mathrm{zl}$, which is comparable in size to proteins and viruses. By comparison, a conventional optical trapping system (with a wavelength almost two times smaller than the operation wavelength of our hybrid device) produces a diffraction limited focal spot that is $\sim 500 \mathrm{~nm}$ in diameter or $\sim 650 \mathrm{fl}$. Although it is possible to trap Rayleigh particles with a conventional trap, in practice the particles will diffuse within this focal volume and not be highly localized. Our hybrid plasmonic-photonic nanoresonator provides a method to not only increase the field gradients, but also to produce a highly localized region with the potential to immobolise nanosized biological particles whilst maintaining a high quality factor for the system (Table 1). This hybrid structure can also be integrated with other photonic devices to create "lab on a chip" devices.

Two conditions must be met to have a successful optical trap for Rayleigh particles. Firstly, the gradient force must be larger than the scattering force. Secondly, the trapping energy must dominate the thermal energy (in other words, the trapping forces must pull the particle in before the Brownian motion of the particle moves it out of the trap). As discussed earlier, the polarizability of the particle in the trap is the key factor in determining how easily it can be trapped (Svoboda and Block 1994). We know that laser induced heating is also proportional to the intensity (Seol et al. 2006) of the light. To trap nanosized particles, the gradient force should be increased while limiting the heating of the sample. For the trapping of biological samples, it is also important to keep the heating to a minimum to maintain the viability of the sample.

\section{Summary}

We have investigated the use of a hybrid photonic-plasmonic nanoresonator for the optical trapping of nanosized biological particles in water. The hybrid structure comprises an $\mathrm{L} 3 \mathrm{PhC}$ cavity based on a GaAs membrane and a BNA. A layer of $\mathrm{SiO}_{2}$ is placed between the two to provide separation and surface passivation. Using FDTD simulations, we demonstrated that the optical intensity in the BNA gap is enhanced by a factor larger than 40 compared to the case when the BNA is not present. We have shown that the optical field is confined in the BNA gap to a volume $\sim 30 \mathrm{zl}$, while the structure maintains a high quality factor (5400-7700)-properties that will aid the development of an efficient optical trap. This hybrid device is also compatible with integrated photonics (such as waveguides and other $\mathrm{PhC}$ devices), increasing its flexibility and making it suitable for a wide range of long term applications in nanotechnology, quantum physics, biology, medicine and chemistry.

Acknowledgments A. J. Wright gratefully acknowledges support from the Royal Academy of Engineering/EPSRC via a personal research fellowship. M. Mossayebi acknowledges the support of the University of Nottingham under the Dean of Engineering scholarship for international excellence. She also thanks Dr. Simeon Kaunga-Nyirenda and Dr. James Bonnyman for their technical support. 
Open Access This article is distributed under the terms of the Creative Commons Attribution 4.0 International License (http://creativecommons.org/licenses/by/4.0/), which permits unrestricted use, distribution, and reproduction in any medium, provided you give appropriate credit to the original author(s) and the source, provide a link to the Creative Commons license, and indicate if changes were made.

\section{References}

Ashkin, A.: Acceleration and trapping particles by radiation pressure. Phys. Rev. Lett. 24, 156-159 (1970)

Ashkin, A.: Optical trapping and manipulation of neutral particles using lasers. Proc. Natl. Acad. Sci. USA 94, 4853-4860 (1997)

Ashkin, A., Dziedzic, J.M., Bjorkholm, J.E., Chu, S.: Observation of a single-beam gradient force optical trap for dielectric particles. Opt. Lett. 11, 288-290 (1986)

Berthelot, J., Acimovic, S.S., Juan, M.L., Kreuzer, M.P., Renger, J., Quidant, R.: 3D manipulation with scanning near-field optical nanotweezers. Nat. Nanotechnol. (2013) arXiv:1311.1740

Bozhevolnyi, S.I., Volkov, V.S., Devaux, E., Laluet, J.-Y., Ebbesen, T.W.: Channel plasmon subwavelength waveguide comonents including interferometers and ring resonators. Nature 440, 508-511 (2006)

Bustamante, C., Bryant, Z., Smith, S.B.: Ten years of tension: single-molecule DNA mechanics. Nature 421, 423-427 (2003)

Cai, H., Poon, A.W.: Optical manipulation and transport of microparticles on silicon nitride microringresonator-based add-drop devices. Opt. Lett. 35, 2855-2857 (2010)

Cetin, A.E., Yanik, A.A., Yilmaz, C., Somu, S., Altug, H.: Monopole antenna arrays for optical trapping, spectroscopy, and sensing. Appl. Phys. Lett. 98, 111110 (2011)

Chen, C., et al.: Enhanced optical trapping and arrangement of nano-objects in a plasmonic nanocavity. Nano Lett. 12, 125-132 (2012)

Chen, Y.-F., Serey, X., Sarkar, R., Chen, P., Erickson, D.: Controlled photonic manipulation of proteins and other nanomaterials. Nano Lett. 12, 16337 (2012)

Cross, S.E., Jin, Y.-S., Rao, J., Gimzewski, J.K.: Nanomechanical analysis of cells from cancer patients. Nat. Nanotechnol. 2, 780-783 (2007)

Deufel, C., Forth, S., Simmons, C.R., Dejgosha, S., Wang, M.D.: Nanofabricated quartz cylinders for angular trapping: DNA supercoiling torque detection. Nat. Methods 4, 223-225 (2007)

Dholakia, K., Reece, P.: Optical micromanipulation takes hold. Nanotoday 1(1), 18-27 (2006)

Dienerowitz, M., Mazilu, M., Dholakia, K.: Optical manipulation of nanoparticles: a review. J. Nanophotonics 2, 021875 (2008)

Friese, M.E.J., Nieminen, T.A., Heckenberg, N.R., Rubinzstein-Dunlop, H.: Optical alignment and spinning of laser-trapped microscopic particles. Nature 394, 348-350 (1998)

Grier, D.G.: A revolution in optical manipulation. Nature 424, 21-27 (2003)

Grigorenko, A.N., Roberts, N.W., Dickson, M.R., Zhang, Y.: Nanometric optical tweezers based on nanostructured substrates. Nat. Photonics 2, 365-370 (2008)

Gustavson, T.L., et al.: Transport of Bose-Einstein condensates with optical tweezers. Phys. Rev. Lett. 88, 020401 (2001)

Juan, M.L., Righini, M., Quidant, R.: Plasmon nano optical tweezers. Nat. Photonics 5, 349-356 (2011)

Khatibzadeh, N., et al.: Determination of motility forces on isolated chromosomes with laser tweezers. Sci. Rep. 4, 6866 (2014)

Kim, B.-S., Nikolovski, J., Bonadio, J., Mooney, D.J.: Cyclic mechanical strain regulates the development of engineered smooth muscle tissue. Nat. Biotechnol. 17, 979-983 (1999)

Ko, K.D., Kumar, A., Fung, K.H., Ambekar, R., Liu, G.L., Fang, N.X., Toussaint Jr., K.M.: Nonlinear optical response from arrays of Au Bowtie nanoantennas. Nano Lett. 11(1), 61-65 (2010)

Liberale, C., Cojoc, G., Bragheri, F., Minzioni, P., Perozziello, G., La Rocca, R., Ferrara, L., Rajamanickam, V., Di Fabrizio, E., Cristiani, I.: Integrated microfluidic device for single-cell trapping and spectroscopy. Sci. Rep. 3, 1258 (2013). doi:10.1038/srep01258

Lin, S., Crozier, K.B.: Planar silicon microrings as wavelength-multiplexed optical traps for storing and sensing particles. Lab Chip 11, 4047-4051 (2011)

Lin, S., Schonbrun, E., Crozier, K.: Optical manipulation with planar silicon microring resonators. Nano Lett. 10(7), 2408-2411 (2010)

Malaguti, S., Bellanca, G., Ottaviano, L., et al.: Tailored design of WDM filters in BCB embedded PhC membranes. Opt. Quantum Electron. 45(4), 329-342 (2013)

Mandal, S., Serey, X., Erickson, D.: Nanomanipulation using silicon photonic crystal resonators. Nano Lett. 10, 99-104 (2010) 
Marag, O.M., Jones, P.H., Gucciardi, P.G., Volpe, G., Ferrari, A.C.: Optical trapping and manipulation of nanostructures. Nat. Nanotechnol. 8 (2013)

Matsuda, T., Sugawara, T.: Control of cell adhesion, migration, and orientation on photochemically microprocessed surfaces. J. Biomed. Mater. Res. 32, 165-173 (1996)

Michael, B., et al.: Nanoassembled plasmonic-photonic hybrid cavity for tailored light-matter coupling. Nano Lett. 10(3), 891-895 (2010)

Min, C., et. al.: Focused plasmonic trapping of metallic particles. Nat. Commun. 4, 2891 (2013). doi:10. 1038/ncomms3891

Misawa, H., Kitamura, N., Masuhara, H.: Laser manipulation and ablation of a single microcapsule in water. J. Am. Chem. Soc. 113, 7859-7863 (1991)

Molloy, J.E., Padgett, M.J.: Lights, action: optical tweezers. Contemp. Phys. 43(4), 241-258 (2002)

Mossayebi, M., Parini, A., Wright, A.J., Somekh, M.G., Bellanca, G., Larkins, E.C.: A realizable hybrid photonic-plasmonic nanocavity with high quality factor and confinement of light. Opt. Express. (2016)(submitted)

Neuman, K.C., Block, S.M.: Optical trapping. Rev. Sci. Instrum. 75, 2787-2809 (2004)

ODell, D., Serey, X., Erickson, D.: Self-assembled photonic-plasmonic nanotweezers for directed selfassembly of hybrid nanostructures. Appl. Phys. Lett. 104, 043112 (2014)

Palik, E.D.: Handbook of optical constants of solids. Academic Press Inc, London (2012)

Pang, Y., Gordon, R.: Optical trapping of a single protein. Nano Lett. 12, 402-406 (2012)

Pauzauskie, P.J., et al.: Optical trapping and integration of semiconductor nanowire assemblies in water. Nat. Mater. 5, 97-101 (2006)

Righini, M., Ghenuche, P., Cherukulappurath, S., Myroshnychenko, V., Garca de Abajo, F.J., Quidant, R.: Nano-optical trapping of Rayleigh particles and Escherichia coli bacteria with resonant optical antennas. Nano Lett. 9(10) (2009)

Roxworthy, B.J., et al.: Application of plasmonic Bowtie nanoantenna arrays for optical trapping, stacking, and sorting. Nano Lett. 12(2), 796-801 (2012)

Renaut, C., et. al.: On chip shapeable optical tweezers. Sci. Rep. (2013)

Sauvan, C., Lalanne, P., Hugonin, J.P.: Slow-wave effect and mode-profile matching in photonic crystal microcavities. Phys. Rev. 71, 165118 (2005)

Schuller, J.A., Branard, E.S., Cai, W., Chul, J.Y., White, J.S., Brongersma, M.L.: Plasmonics for extreme light concentration and manipulation. Nat. Mater. 9(3), 193-204 (2010)

Seol, Y., Carpenter, A.E., Perkins, T.T.: Gold nanoparticles: enhanced optical trapping and sensitivity coupled with significant heating. Opt. Lett. 31(16) (2006)

Svoboda, K., Block, S.M.: Optical trapping of metallic Rayleigh particles. Opt. Lett. 19(13) (1994)

Taflove, A., Hagness, S.C.: Computational electrodynamics: the finite-difference time-domain method, 3rd edn. Artech House, Boston (2005)

Van Leest, T., Caro, J.: Cavity-enhanced optical trapping of bacteria using a silicon photonic crystal. Lab Chip 13, 4358-4365 (2013)

Wong, H.M.K., et al.: On-a-chip surface plasmon tweezers. Appl. Phys. Lett. 99, 061107 (2011)

Yao, A.M., Padgett, M.J.: Orbital angular momentum: origins behavior and applications. Adv. Opt. Photonics 3(2), 161-204 (2011)

Yu, S.-P., Hood, J.D., Muniz, J.A., Martin, M.J., Norte, R., Hung, C.-L., Meenehan, S.M., Cohen, J.D., Painter, O., Kimble, H.J.: Nanowire photonic crystal waveguides for single-atom trapping and strong light-matter interactions. Appl. Phys. Lett. 104, 111103 (2014)

Zhang, W., et al.: Trapping and sensing $10 \mathrm{~nm}$ metal nanoparticles using plasmonic dipole antennas. Nano Lett. 10, 315201 (2010)

Zhang, T., Callard, S., Jamois, C., Chevalier, C., Feng, D., Belarouci, A.: Plasmonic-photonic crystal coupled nanolaser. Nanotechnology 25(31), 315201 (2014)

Zhong, M.-C., Wei, X.-B., Zhou, J.-H., Wang, Z.-Q., Li, Y.-M.: Trapping red blood cells in living animals using optical tweezers. Nat. Commun. 4 (2013) 